\title{
Thermographic Findings in Patients with Lumbar Spinal Stenosis Before and After Walking
}

\author{
Tae Shin Kim, Junseok W Hur, Seok Jin Ko, Ju Kyung Shin, Jung Yul Park \\ Department of Neurosurgery, Korea University Anam Hospital, Korea University College of Medicine, Seoul, Korea
}

\begin{abstract}
Objective: Patients with lumbar spinal stenosis experience a variety of symptoms, including back pain, and neurogenic claudication. However, these symptoms appear only after walking for a certain distance. Therefore, it is difficult to make an accurate diagnosis using conventional methods, which involve tests performed under resting conditions. Therefore, infrared thermography could be a helpful diagnostic tool. Methods: Eight patients, who had neurogenic claudication after walking a certain distance, were enrolled in this study. These patients underwent infrared thermography at least twice after they walked a certain distance (about 50 meters after walking for 10 minutes). Four other patients, who only had back pain but no claudication, were enrolled as the comparison groups. Results: Seven patients in the patient group showed a decrease of 0.6 to 2.3 degrees in body temperature, depending on the body regions evaluated, after walking a certain distance. However, in the comparison groups, only 1 patient had decreased body temperature after walking. Conclusion: Neurogenic claudication symptoms are present in spinal stenosis patients because of venous congestion, which causes neurogenic claudication and decreases surface temperature. Therefore, infrared thermography can be performed in patients not only in resting conditions but also in symptom-expression conditions. Hence, infrared thermography can help in the accurate diagnosis of lumbar spinal stenosis.
\end{abstract}

Key Words: Infrared; Thermography; Spinal stenosis; Claudication

$\triangle$ Corresponding Author: Jung Yul Park, Department of Neurosurgery, Korea University Anam Hospital, Korea University College of Medicine, 73 Inchon-ro, Seongbuk-gu, Seoul 02841, Korea. Tel: +82-2-920-5391, 3491, Fax: +82-2-920-0629, E-mail: jypark98@korea.ac.kr

\section{INTRODUCTION}

Patients with lumbar spinal stenosis complain of symptoms, such as back pain, lower extremity radiating pain, and neurogenic claudication. However, many patients have no distinguishing symptoms at resting state, but symptoms appear only after patients start walking for a certain distance. Therefore, it may be difficult to make an accurate diagnosis by using conventional methods, which are performed only under resting positions.

The skin is the largest organ in the human body, and the blood flow to the skin fluctuates. Skin function is influenced by several diseases, such as hypertension ${ }^{3)}$, genetic discordances of vascular reactivity ${ }^{19}$, and peripheral vascular tone ${ }^{13)}$. Skin temperature is affected by vasomotor activity, which is regulated by the peripheral sensory nerve fibers? ${ }^{6}$. Infrared thermography has been used for the measurement of skin temperature in musculoskeletal disorders ${ }^{4}$. Particularly, infrared thermography is useful for the diagnosis and screening of neuropathy in patients with low back pain. The purpose of this study was to determine whether infrared thermography could be used to diagnose lumbar spinal stenosis through an analysis of changes in skin temperature after patients walk for a certain distance.

\section{MATERIALS AND METHODS}

A total of eight patients diagnosed with lumbar spinal stenosis were enrolled in this study. All patients complained of radiating pain, low back pain, or other symptoms (such as paresthesia) in resting conditions, and neurogenic claudication after walking for a certain distance. Patients with diabetes, heart disease, peripheral circulation disorders, or peripheral neuropathy were excluded in this study. For these patients, skin temperature was measured with infrared thermography. Thermographic tests were done at least twice for these patients, in resting state and in a condition when neurogenic claudication appeared after walking for a certain distance. The results were correlatively analyzed with the clinical symptoms of patients.

Other four patients were selected for the comparison groups. These four patients were divided two groups: one group included two patients who were very old and complained of only back pain; another group included other two patients who had no back pain. All of these four patients had neurogenic claudication after walking for a certain distance. The infrared thermography results were analyzed and compared in these groups. 


\section{RESULTS}

Patients with spinal stenosis included 7 men and 1 women. The mean age at admission was 71.3 years, and ranged from 58 to 84 years (Table 1). All patients complained of neurogenic claudication after walking for a certain distance (about 50 meters after a 10-minute walk). Low back pain was present in 6 patients but not in the other 2 patients. Five patients presented with bilateral neurogenic claudication, and 3 patients had unilateral neurogenic claudication. The mean duration of symptoms was 16.8 months, and ranged from 3 months to 5 years.

Seven individuals in the patient group showed a decrease

Table 1. Characteristics of 8 spinal stenosis patients who underwent infrared thermographic imaging

\begin{tabular}{ll}
\hline \hline Characteristics & \multicolumn{1}{c}{ Values } \\
\hline Median age (range) & $\begin{array}{l}71.3 \text { years } \\
(58-84 \text { years) }\end{array}$ \\
\hline Sex (\%) & $7(87.5)$ \\
\hline Male & $1(12.5)$ \\
\hline Female & $6(75)$ \\
\hline Low back pain (\%) & $5(62.5)$ \\
Bilateral neurogenic claudication (\%) & $3(37.5)$ \\
Unilateral neurogenic claudication (\%) & 16.8 months \\
\hline Mean duration of symptoms (range) & $(3$ months-5 years) \\
\hline
\end{tabular}
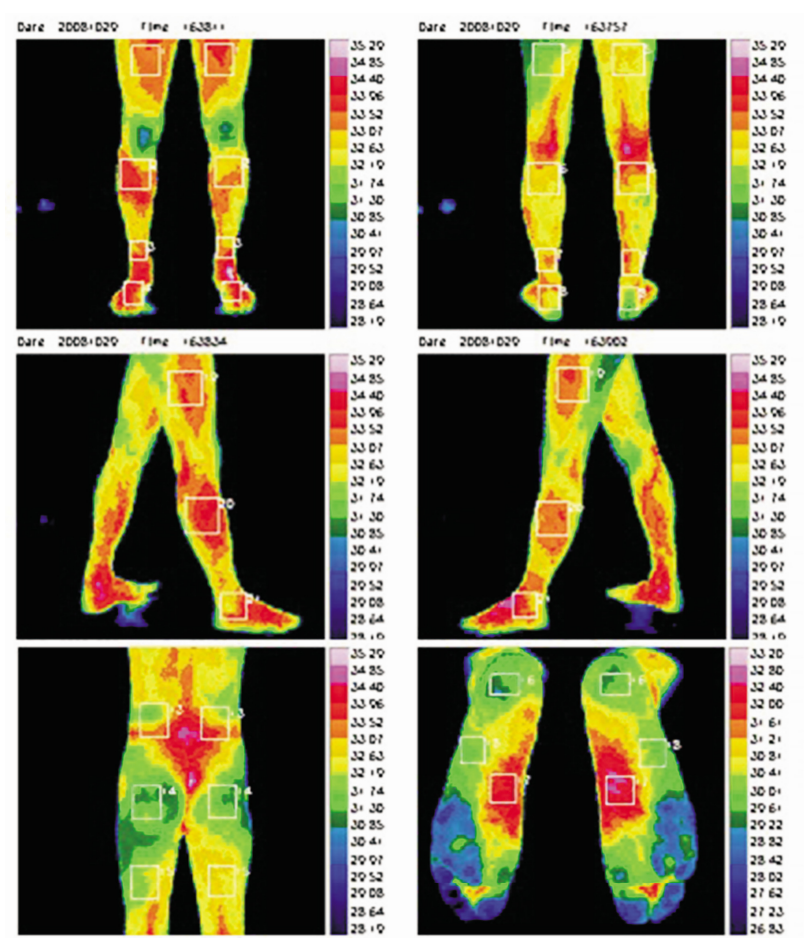

Baseline of 0.6 to 2.3 degrees in body temperature, depending on the body regions examined, after walking for a certain distance (Fig. 1, Table 2). However, the extent of body temperature reduction was different in different individuals. In the comparison group, the body temperature increased by 1.5 to 5 degrees in the lumbar region and lower extremities in 2 patients, decreased in 1 patient, and did not change in another patient after 10 minutes of walking (Fig. 2). The alteration in skin temperature after walking was relatively consistent with the expression of symptoms, although quantitative analysis and comparisons were not performed in this study. No body temperature changes were observed in chronic low back pain patients who did not have neurogenic claudication.

Table 2. Surface temperature changes in the patient and comparison groups

\begin{tabular}{|c|c|}
\hline Characteristics & Values (\%) \\
\hline \multicolumn{2}{|c|}{$\begin{array}{l}\text { Surface temperature in stenosis patients } \\
\text { after 10-minute walking }\end{array}$} \\
\hline Increased & $0(0)$ \\
\hline Decreased & $7(87.5)$ \\
\hline Unchanged & $1(12.5)$ \\
\hline \multicolumn{2}{|c|}{$\begin{array}{l}\text { Surface temperature in the comparison group } \\
\text { after 10-minute walking }\end{array}$} \\
\hline Increased & $2(50)$ \\
\hline Decreased & $1(25)$ \\
\hline Unchanged & $1(25)$ \\
\hline
\end{tabular}
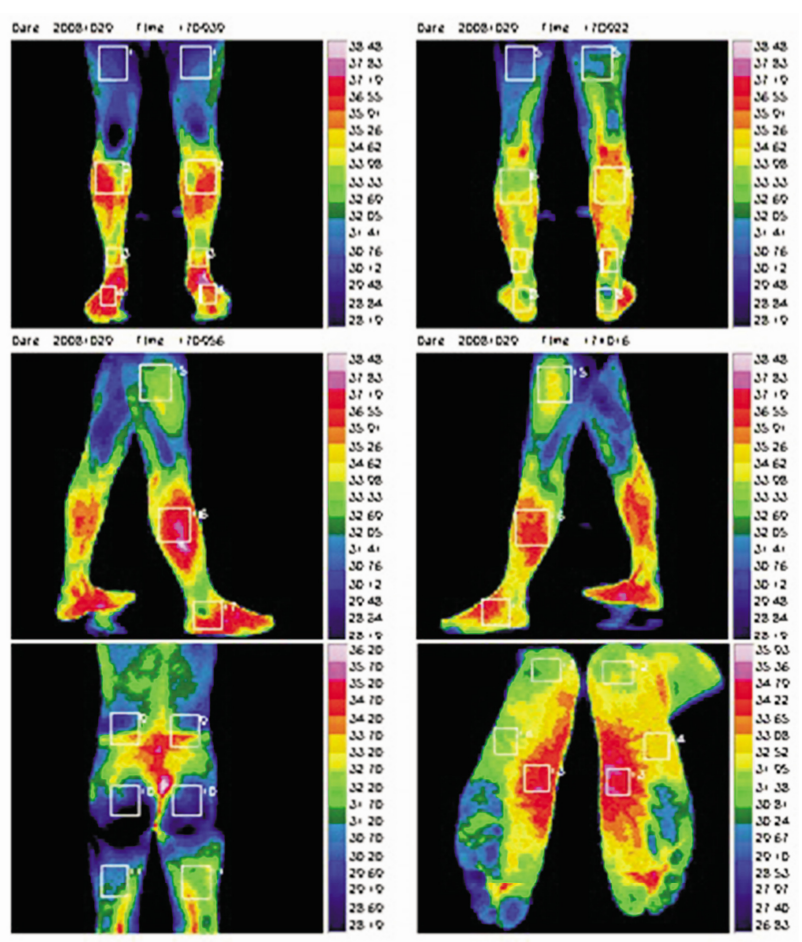

After Walking

Fig. 1. Comparison of thermographic images of a patient with lumbar stenosis and a symptom of claudication before and after walking. 

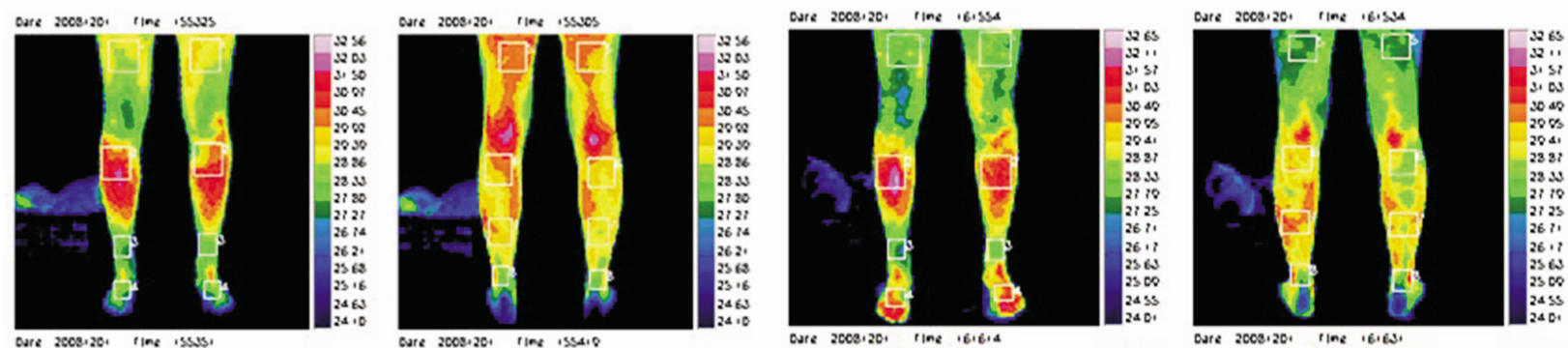

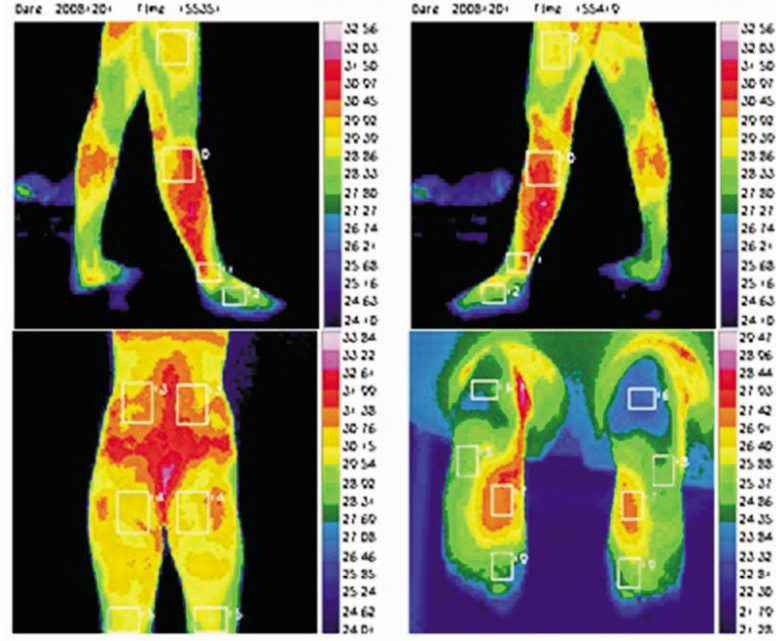

Baseline

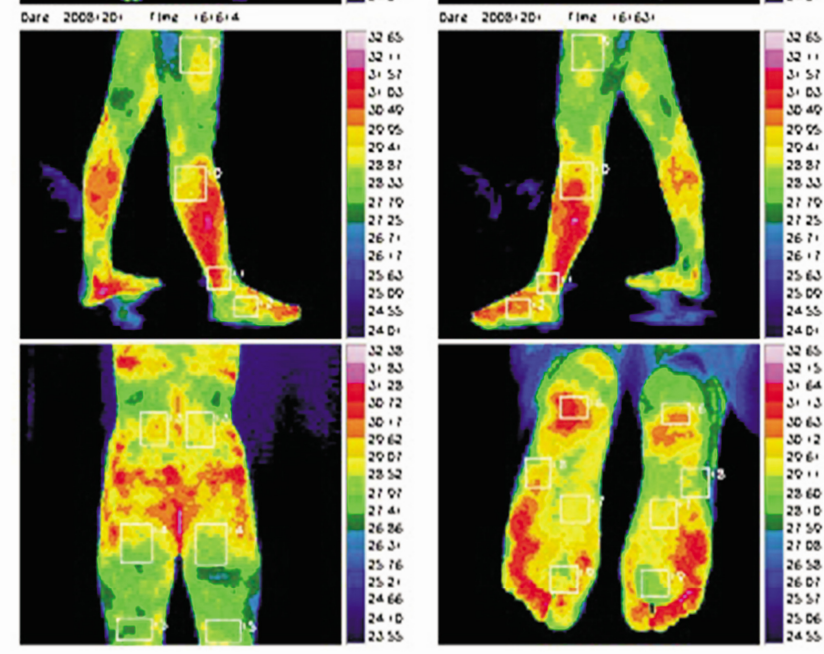

After Walking

Fig. 2. Comparison of thermographic images of a patient in comparison group before and after walking.

\section{DISCUSSION}

Claudication symptoms cause a decline in body surface temperature in regions affected by pain in patients with spinal stenosis. Neurogenic claudication symptoms occur in spinal stenosis patients because when the spinal root is compressed, venous congestion occurs, and the inadequate drainage reduces oxygenation or increases the accumulation of metabolites in the cauda equina ${ }^{14)}$. Reduced oxygenation results in ischemia, which contributes to development of neurogenic claudication ${ }^{2)}$. Transient ischemia often occurs during walking because of the increased oxygen demand ${ }^{2}$. Hypoxic ischemia can result in decreased surface body temperature ${ }^{12}$. Infrared thermography can promptly measure a decrease in skin temperature. Therefore, infrared thermography can be used to visualize the temperature changes that occur after claudication symptoms appear. Magnetic resonance imaging (MRI) can be used to visualize static symptoms in patients under resting conditions, but infrared thermography can reveal dynamic changes in patients in a convenient manner.

Patients with lumbar spinal stenosis show several symptoms, such as low back pain, lower extremity radiating pain, and claudication. However, these symptoms sometimes do not appear under resting conditions and can be observed only after the patients walk for a certain distance. Therefore, it is difficult to make an accurate diagnosis using conventional methods, which involve tests that are performed only under resting conditions.

The skin surface radiates infrared rays in a wavelength range of 900-14,000 nanometers, and infrared thermography can be used to detect this infrared radiation. Subsequently, infrared thermography can be used to produce a visible two-dimensional image of the distribution of the skin surface temperatures of the human body ${ }^{20)}$. Infrared thermography was first used in the medical field in $1956^{10}$. Thereafter, many related studies have been performed in several fields, such as mammology, rheumatology, orthopedics, neurology, and vascular imaging, and for different types of screenings or physiological response examinations ${ }^{8,15)}$. Infrared thermography, suitable for objective visualization of skin temperature, is a valuable and non-invasive technique for diagnosis. The pathological examination of spinal stenosis is another potential use of infrared thermography. Abert et al. ${ }^{1)}$ first used infrared thermography to measure skin temperature in low back pain patients. Subsequently, several related reports have been published. For example, some studies used a highly sensitive infrared thermography protocol to show that skin temperature abnormalities were associated with spinal radiculopathy ${ }^{5,9,16-18)}$. The findings confirm that the results obtained using infrared thermography are well correlated with those obtained with computerized tomography (CT), myelography, and 
MRI. Moreover, Hakelius et al. ${ }^{7)}$ and Lindholm et al. ${ }^{11)}$ commented that low temperature measured in the lower extremities indicated the possibility of spinal nerve root compression.

Symptoms do not explicitly appear in many cases of lumbar spinal stenosis. CT or MRI is performed under resting conditions, where the symptoms are not present; therefore, it is difficult to correlate the patient's symptoms with the findings obtained with CT or MRI. Moreover, it is also difficult to choose a treatment plan with the results from other medical techniques, such as electromyography and examination of somatosensory evoked potentials. In this study, we could directly identify actual lesions using infrared thermography by measuring changes in body temperature when the patient's symptoms appeared. Infrared thermography can help in designing a detailed treatment plan based on the identification of the actual lesions, which are difficult to detect using CT or MRI alone. Another feature of infrared thermography is that the therapeutic effects can be easily observed. Currently, we are examining the effect of treatment by investigating the changes in subjective symptoms after conservative treatments, such as Nerve block, radiofrequency ablation, or surgery. Through a comparison of the results obtained before and after surgery using infrared thermography, objective conclusions regarding the effects of the surgery can be obtained. The symptoms that patients complain are the most important indicator for the diagnosis but more advanced diagnosis techniques are needed for the management of patients.

Our study has several limitations. First, the number of patients was too small to perform statistical analyses. Second, infrared thermography before walking was performed in a standardized environment, but infrared thermography after walking was done not in stable position. Therefore, the test conditions before and after walking were different.

\section{CONCLUSION}

In this study, infrared thermography was used to measure skin temperature in patients, not only under resting conditions but also under conditions where symptoms appear. Infrared thermography can help in the accurate diagnosis of lumbar spinal stenosis. However, further studies are needed in order to determine the practical usefulness of infrared thermography, to reveal correlations between the severity of symptoms and body temperature, and to standardize the test conditions.

\section{REFERENCES}

1. Albert SM, Glickman M, Kallish M: Thermography in orthope- dics. Ann N Y Acad Sci 121:157-170, 1964

2. Alvarez JA, Hardy RH: Lumbar spine stenosis: a common cause of back and leg pain. Am Fam Physician 57:1825-1834; 18391840, 1998

3. Boutcher YN, Park YJ, Boutcher SH: Vascular and baroreceptor abnormalities in young males with a family history of hypertension. Eur J Appl Physiol 107:653-658, 2009

4. Campero M, Verdugo RJ, Ochoa JL: Vasomotor innervation of the skin of the hand - a contribution to the study of human anatomy. J Anat 182:361-368, 1993

5. Gillstrom P: Thermography in low back pain and sciatica. Arch Orthop Trauma Surg 104:31-36, 1985

6. Gold JE, Cherniack M, Hanlon A, Dennerlein JT, Dropkin J: Skin temperature in the dorsal hand of office workers and severity of upper extremity musculoskeletal disorders. Int Arch Occup Environ Health 82:1281-1292, 2009

7. Hakelius A, Nilsonne U, Pernow B, Zetterquist S: The cold sciatic leg. Acta Orthop Scand 40:614-623, 1969

8. Jones BF: A reappraisal of the use of infrared thermal image analysis in medicine. IEEE Trans Med Imaging 17:1019-1027, 1998

9. LaBorde TC: Thermography in diagnosis of radiculopathies. Clin J Pain 5:249-253, 1989

10. Lawson R: Implications of surface temperatures in the diagnosis of breast cancer. Can Med Assoc J 75:309-311, 1956

11. Lindholm RV, Myllyla T, Sarvaranta J: The cold foot symptom in sciatica. A clinical and thermographic study. Ann Chir Gynaecol 70:176-181, 1981

12. Manley DM, Xiang B, Kupriyanov VV: Visualization and grading of regional ischemia in pigs in vivo using near-infrared and thermal imaging. Can J Physiol Pharmacol 85:382-395, 2007

13. Nagashima K, Yoda T, Yagishita T, Taniguchi A, Hosono T, Kanosue K: Thermal regulation and comfort during a mild-cold exposure in young Japanese women complaining of unusual coldness. J Appl Physiol 92:1029-1035, 2002

14. Porter RW: Spinal stenosis and neurogenic claudication. Spine 21:2046-2052, 1996

15. Ring EF: The historical development of thermal imaging in medicine. Rheumatology 43:800-802, 2004

16. Takahashi Y, Takahashi K, Moriya $\mathrm{H}$ : Thermal deficit in lumbar radiculopathy. Correlations with pain and neurologic signs and its value for assessing symptomatic severity. Spine 19:2443-2449; discussion 2449-2450, 1994

17. Thomas D, Cullum D, Siahamis G, Langlois S: Infrared thermographic imaging, magnetic resonance imaging, CT scan and myelography in low back pain. Br J Rheumatol 29:268-273, 1990

18. Uematsu S, Jankel WR, Edwin DH, Kim W, Kozikowski J, Rosenbaum A, et al.: Quantification of thermal asymmetry. Part 2: Application in low-back pain and sciatica. J Neurosurg 69:556561, 1988

19. Wu T, Snieder H, de Geus E: Genetic influences on cardiovascular stress reactivity. Neurosci Biobehav Rev 35:58-68, 2010

20. Zaproudina N, Airaksinen O, Narhi M: Are the infrared thermography findings skin temperature-dependent - a study on neck pain patients. Skin Res Technol 19:e537-544, 2013 